\title{
Tuberculosis Renal en Pediatría
}

\author{
Dr. Waldo Rodríguez Y. ${ }^{1}$; Dr. Eduardo Caffarena A.2,4; Dra. María Antonia Bidegaín S. ${ }^{3}$; \\ Dra. Sonia Figueroa Y. 1; Dra. M. Soledad Rodríguez T, 1; Dr. Alfredo Pugh M.4
}

\section{Renal Tuberculosis in Pediatrics}

Sixteen clinical records of patients 6 to 15 years old with renal tuberculosis registered over a 21 years period, were analy sed. An increased incidence of this condition in the last 8 years was observed. Macrohematuria (56.3\%) and enuresis (18.8\%) were the furst evidences of the disease. Radiological findings at the excretory urography, corresponded to grades 0 , in four patients, I in six and JI through IV in four children (Lattimer's modificd classification). Lesions were of grades II or less, in $75 \%$ of the cases. In all patients the diagnosis was confirmed either by the isolation of tubercle bacilli from urine, as ocurred in 14 patients (87.5\% or by distinctive histological findings in the other two cases. The results of treatment were satisfactory in eleven properiy followed cases (68.7\%). Three patients were followed up in different health centers and information of their final state was not available for us. The scarcity of clinical symptoms and radiological signs in the reported patients is attributed to early diagnosis and an adequate management.

(Key words: Tuberculosis. Renal. Macrohematuria, Aseptic pyuria. Urographic abnormalities).

La frecuencia de tuberculosis en el niño $y$ adolescente menor, ha disminuido en los uiltimos atios, a diferencia de lo que ocurre en el adulto. En Chile, durante el año 1983, se notificaron 6.989 casos de tuberculosis, 403 de los cuales correspondieron a menores de 15 atios $(5,8 \%)$.

1. Departamento de Pediatría, Facultad de Medicina, Universidad de Concepción.

2. Sección Urología. Depto. Cirugía. Facultad de Medicina. Universidad de Concepción.

3. Servicio de Pediatría. Hospital Guilermo Grant B. de Concepción.

4. Servicio de Urología, Hospital Guiliermo Grant B. de Concepción.
De los 1.134 casos $(16,2 \%)$ de la Octava Región, un $19,1 \%$, corresponde a tuberculosis extrapulmonar $^{1}$. De 553 casos $(7,9 \%)$ de Concepción, $21,8 \%$ corresponde a tuberculosis extrapulmonar $^{1}$. En países con diferentes niveles de vida $y$ en el tnuestro, los pacientes con tuberculosis renal en $\mathbf{l}$ edad pediátrica son escasísimos $1,2,3,4,5$, 6.

El aparente aumento de la incidencia de esta patología en nuestro medio, nos ha motivado a relatar nuestra experiencia.

\section{MATERIAL y METODO}

Se estudiaron 16 pacientes, en quienes se formuló el diagnóstico de tuberculosis renal, controlados en la 
Policlinica de Nefrología del Servicio de Pediatrja del Hospital Guilermo Grant Benavente de Concepción, en el período comprendido entre Enero de 1964 y Diciembre de 1984. Cuatro pacientes fueron estudiados entre los años 1964 y 1974, y 8 entre $1975^{\circ}$ y 1984 . Tres pacientes procedían de Concepción urbano, 4 de Talcahuano y los restantes de diferentes localidades de las regiones Octava y Novena del país.

El estudio se realizó a todo niño y adolescente que presentó hematuria, piuxia aséptica o ambas. El diagnóstico fue confirmado mediarte estudios microbiológicos, histológicos o ambos. Desde 1978, se efectúa como mínimo 6 baciloscopias y cultivos de orma, con un máximo de 12, a cada paciente. En la clasificación de las alteraciones radiológicas, se usó el criterio de Lattimer modificado ${ }^{7,8}$ (Tabla 1).

Tabla 1.

Clasificación Radiológica de la Tuberculosis Renal según Lattimer

\begin{tabular}{ll}
\hline Grado 0: & $\begin{array}{l}\text { Urografía excretora nomal. } \\
\text { Grado I: }\end{array}$ \\
Grado II: & $\begin{array}{l}\text { Pequerniento y/o irregularidad de papila. } \\
\text { tadas en una zona del rinón. }\end{array}$ \\
Grado IIJ: & $\begin{array}{l}\text { Grandes cavernas y/o dilataciones. Urete- } \\
\text { ritis. }\end{array}$ \\
Grado IV: & $\begin{array}{l}\text { Lestones destructivas en más de } 3 / 4 \text { partes } \\
\text { del rinón. Lesiones estenóticas de uréter. } \\
\text { Retracción vesical. Exclusión Ienal. }\end{array}$
\end{tabular}

Nota: Los grados III y IV se consideran avanzados.

\section{RESULTADOS}

La procedencia de los enfermos fue urbana; tres de Concepción, 4 de Talcahuano y los demás de diversas localidades de la Octava y Novena regiones. Su edad fluctuó entre 6 y 15 años, promedio de 10 años y 3 meses; la mayoría se agrupó entre los 8 y 15 años de edad $(81,2 \%)$, sin diferencia significativa entre ambos sexos. Dos pacientes no recibieron vacuna BCG en el período de recién nacido; cuantro tuvieron contactos tuberculosos intrafamiliares. Un paciente había sido operado de absceso perirrenal; otro niño habia recibido tratamiento por tuberculosis pulmonar 6 años antes de detectarse la afección renal y otro habia recibido tratamiento profiláctico con isoniacida a los 18 meses de edad por contacto con paciente tuberculoso.

El motivo de consulta de 9 pacientes $(56,3 \%)$ fue macrohematuria; en 3 enuresis (18.8\%); y en un paciente en cada caso por síndrome nefrítico agudo con hematuria persistente, uno por retención urinaria, dolor de fosa lumbar y disuria con polaquiuria respectivamente. En general los síntomas fueron inespecificos durante la evolución. Diez pacientes $(62,5 \%)$ evolucionaron con hematuria macroscópica (en 5, coágulos); 9
$(56,3 \%)$ con disuria; $8(50 \%)$ con polaquiuria $y, 7$ $(43,8 \%)$ con enuresis. En el examen físico se detecto retardo pondoestatutal en 6 pacientes; astenia y adinamia en 3 ; fiebre en 2 ; hipertensión en 2; globo vesical en uno y engrosamiento epididimario en otro.

El examen citoquímico de orina mostró microhematuria en 12 niños (75\%) y piuria aséptica en $11(68,8 \%)$. Sólo 5 pacientes tenian proteinuria mayor de $1 \mathrm{~g} \times 1$, no atribuible a la macrohematuria, que desapareció con el trata. miento. La radiografía de tórax fue nornal en 9 pacientes; en 2, se observaron lesiones minimas calcificadas; en uno un ganglio calcificado y en otro, tuberculosis pulmonar activa. E1 hemogra. ma mostró anemia normocrómica en 6 casos, con valores de hemoglobina inferior a $7,5 \mathrm{~g} . \%$, en 4 de ellos; leucocitosis, en 6 pacientes, superior a 20.000 por $\mathrm{mm}^{3}$, en uno. La eritrosedimentación estaba elevada en 9 niños, en 3 era mayor que $60 \mathrm{~mm}$ en la primera hora.

La función renal era normal en todos los pacientes, en el momento del diagnóstico. La reacción de tuberculina fue superior a $10 \mathrm{~mm}$ de diámetro, sólo en 4 pacientes.

La urografía excretora resultó normal en 4 pacientes (grado 0); en 6 era grado l; en 2 grado II; en uno grado III y grado IV en 3 casos. Estos últimos eran pacientes diagnosticados tardiamen. te (Figura 1). Sólo en tres enfermos se realizó cistouretrografía miccional. En uno fue normal; en uno hab́a reflujo vésicoureteral de grado IV y en otro estenosis uretral.

La búsqueda del bacilo de Koch en la orina de los 16 pacientes, dio resultados positivos en 14 $(87,5 \%)$ con histología positiva en dos de ellos. En un caso, con resultados negativos en las baciloscopias y cultivos, la histología fue característica y en otro, el diagnóstico se certificó con baciloscopía e histología positiva, pero los cultivos de Koch fueron reiteradamente negativos. En 9 casos hubo concordancia entre baciloscopía y cultivos; y, en 5 la baciloscopía fue negativa con cultivos positivos.

En nuestros pacientes, la mayoría de los resultados bacteriológicos positivos fueron obtenidos después de tres baciloscopías y entre el tercero y quinto intento de cultivo. En un paciente el resultado positivo se obtuvo sólo en el séptimo cultivo. El estudio del bacilo de Koch en el contenido gástrico se realizó en 8 pacientes y fue positivo sólo en un paciente con tuberculosis pulmonar activa.

En 11 pacientes $(68,8 \%)$ se hizo sólo tratamiento médico, en $5,(32,2 \%)$ médico-quinúrgico. En $10(62,5 \%)$ se empleó el esquema clásico de 18 meses de duración ${ }^{12,13}$ y en 6 , el esquema abreviado de 6 meses $^{9}$. De estos últimos, 2 

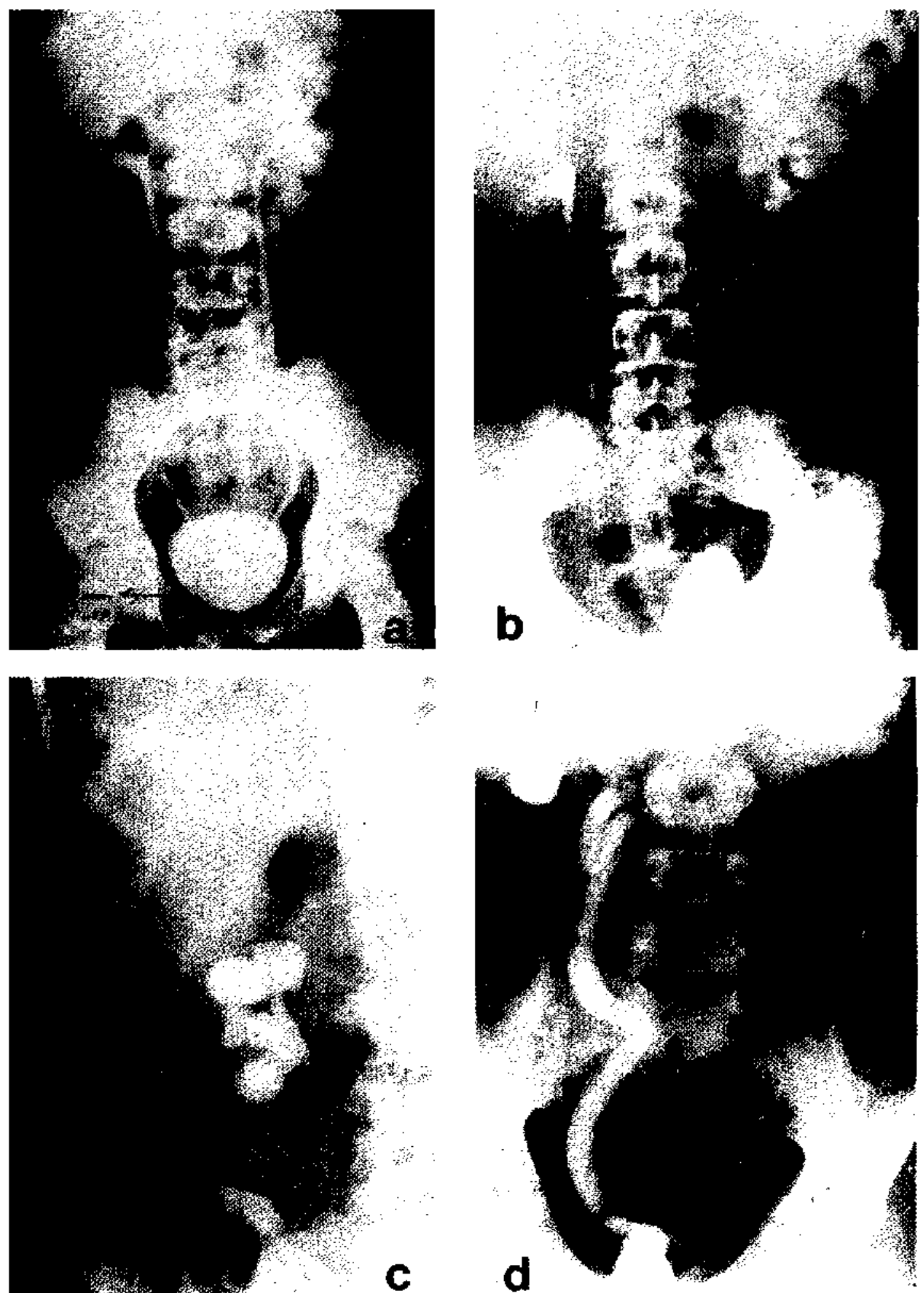

Figura 1. Alteraciones radiológicas según clasificación de Lattimer modificada: a) Grado I; papilitis cálices derechos: b) Grado If: retracción cuellos caliciares a hidrocalicosis izquierda; c) Grado III: riñón mastic derecho (urografía ascendente); đ) Grado IV: retracción vesical con hidrouréteronefrosis, en paciente monorreno izquierdo. 
sufrieron recaidas que fueron tratadas nuevamente con el mismo esquema, con buen éxito esta vez. De los 5 niños que requirieron manejo quirúrgico, 4 sufrieron nefrectomias y uno cistostomía y dilatación uretral. Al cabo de un año de evolución un paciente nefrectomizado debió someterse a nefrostomia de descarga, ileocistoplastía y reemplazo de la terapia clásica por drogas antituberculosas de segunda línea. La evolución fue satisfactoria en 11 pacientes; según criterios microbiológicos y urográficos de curación. En los restantes se desconoce la evolución, pues fueron devueltos al hospital de origen.

\section{COMENTARIO}

La tuberculosis renal en el niño y adolescente menor es considerada de muy baja incidencia en el mundo y ha sido poco descrita en nuestro país. Sin embargo, en la Octava Región debe tenerse presente en casos con hematurias urológicas y piurias asépticas. El notorio aumento de la detección de casos en los últimos 8 años puede ser atribuido en parte, a la investigación sistemática del bacilo de Koch en la orina mediante dos series de 6 muestras cada una, en los años más recientes, en casos con hematuria y piuria aséptica.

Desde el punto de vista epidemiológico desta. can la procedencia urbana de los pacientes, la ausencia de antecedentes de enfermedad tuberculosa previa (salvo un caso) y el antecedente de contacto tuberculoso intra-familiar en una proporción importante de los pacientes.

La patogenia de la tuberculosis genitourinaria se explicaría por diseminación hematógena del bacilo desde focos primarios activos o inaparentes, tratados o curados en forma espontánea $2,5,6,9$ (Figura 2), la que ocurriría precozmente, antes del periodo alérgico, localizándose el bacilo en la corteza renal meses o años antes de la expresión clínica de la enfermedad del riñón.

La macrohematuria constituyó en nuestra serie el principal motivo de consulta. Por otra parte algunos pacientes que consultaron por enuresis fueron manejados previamente como problemas psicopatológicos, sin realizar como norma, exámenes de orina.

La evaluación de la enferme dad confirmó que, aún cuando, la reacción tuberculínica sea positiva en un número limitado de pacientes $(25 \%)$, la búsqueda del bacilo de Koch en la orina resulta positiva en la mayoría de ellos y en los restantes el diagnóstico puede ser confirmado histológicamente. La mayor frecuencia de identificación del bacilo en cultivos de tercera a quinta muestras de orina, e incluso en la séptima, se explicaría por la escasa y cíclica eliminación de

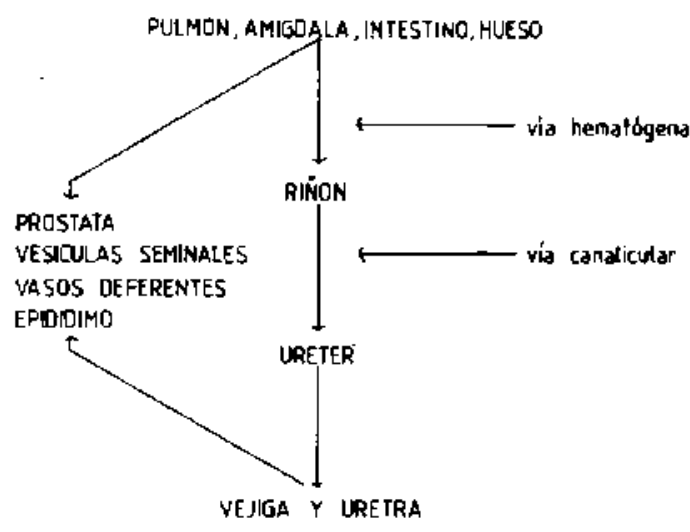

- urology, votumen 20 n2, agosto 1982

Figura 2. Patogenin de la tuberculosis genitourinaria.

gérmenes por la orina ${ }^{2}$, justificando plenamente la norma de solicitar dos series de 6 muestras de orina cada una.

Los estudios radiológicos, mostraron lesiones de grado avanzado en pocos pacientes, todos antes de esta década. La presencia de un númeto importante de sujetos con rifones normales o con lesiones mínimas, podría guardar relación con el diagnóstico precoz. No observamos las calcificaciones descritas por otros autores en las urografias excretoras $2,10,11$.

El diagnóstico definitivo de tuberculosis renal requiere demostrar el bacilo de Koch en la orina. aunque el resto de la evidencia clínica sea muy sugerente. Sin embargo, ocasionalmente, la clínica puede ser suficiente para justificar la iniciación de la terapia antituberculosa, antes de conocer el resultado bacteriológico.

Basados en nuestra experiencia, podemos sugerir que la investigación del bacilo de Koch en la orina de todo niño o adolescente con hematuria urológica o piuria aséptica, permitirá reconocer oportunamente a la tuberculosis renai, lo que es de gran importancia pues, aún si el tratamiento antituberculoso es adecuado, en los grados más avanzados de la enfermedad el proceso de cicatrización y fibrosis continúa y puede obligar al empleo de cirugía mutiladora, como observamos en nuestros primeros pacientes.

\section{RESUMEN}

Se analizaron 16 observaciones clínicas de pacientes con tuberculosis renal, de 6 a 15 años de edad, en un período de 21 años. La frecuencia con que se identificó la enfermedad aumentó en los últimos 8 años. La mayoría de los pacientes era de procedencia urbana. La macrohematuria y 
la enuresis fueron los principales motivos de consulta. En la urografía excretora los hallazgos radiológicos correspondían a los grados 0 , I y II de la clasificación de Lattimer modificada, en el $75 \%$ de los casos. En todos los pacientes el diagnóstico fue confirmado: por la identificación de bacilo de Koch en la orina (entre el $3^{\circ}$ y $5^{\circ}$ cultivo) en $87,5 \%$ de los casos e histología característica en el $12,5 \%$ de los casos. La respuesta al tratamiento fue satisfactoria en los pacientes que completaron el seguimiento de centrol.

\section{REFERENCIAS}

1. Anuario 1983: Enfemedades de notificación obligatoria. Ministerio de Salud. Departamento de Control y Evaluación. Diciembre 1984. Chile.

2. Cos L.R., Cockett A.T.: Genitourinary tuberculosis revisted. Urology, 20: $111,1982$.

3. Editorial: Genitourinary tuberculosis. Brit. Mid. J. 2: 183,1971 .

4. Cooper H.B., Robinson $E_{.} G_{.}$. Treatment of genitourisary tuberculosis: report after 24 years. J.
Urol. 108: 136, 1972.

5. Wisnia L., Lopez de Santa María J., Kukob S.: Problemas diagnósticos en TBC urogenital: estudio de 135 pacientes. Rev. Med. Chil. 104: 77, 1976.

6. Ehrich R.M., Lattimer J.K.: Urogenital tubetculosis in children. J. Urol, 105: 461, 1971.

7. Campbel's Urology: Genitourinary tuberculosis. Lattimer, J.K.; and Wechsler, 4a. Ed. Ed. W.B. Saunders Co., Philadelphia, 1978, Vol. 1 Pág. 557.

8. Vargas $R$ : Urología y afecciones genitales masculinas. Ed. Lniversitaria, Santiago, Chile, 1979, Pág. 557.

9. Narayand A.S.: Overwiew of renal tuberculosis. Urology 19: 231, 1982.

10. Black D., Jones N.F.: Renal Disease. 4th. Ed., Blackwell, 1979, Pág. 249.

11. Smith G.: Urología General. 7a. Ed. Manual Moderno 1983, México, Pág. 179.

12. Siman H., Weinstein B., Pasternak M., Swartz M., Kunz L.: Genitourinary tuberculosis: Clinjcal Fealures in a General Hospital Population. Am. J. Med. $63: 410,1977$.

13. Lattimer J.K., Wechster M., Ehrich R. Fukushima $K$.: Current treatment for renal tuberculosis. J. Urol. 102: 2, 1969. 